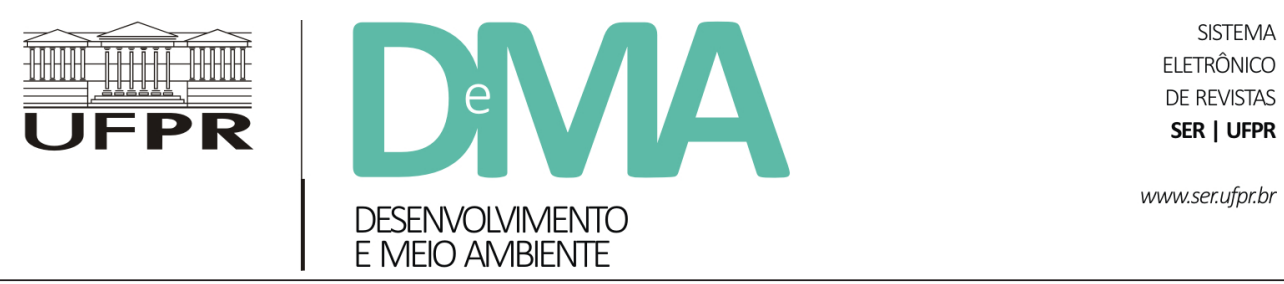

\title{
Métodos para avaliar efetividade de gestão: o caso particular das Áreas de Proteção Ambiental (APAs)
}

\section{Evaluating the Management Effectiveness: The Specific Case of Environmental Protection Areas (APAs)}

\author{
Laura Dias PRESTES ${ }^{1 *}$, Luís Fernando Carvalho PERELLO² ${ }^{2}$ Nelson Luiz Sambaqui GRUBER ${ }^{3}$ \\ ${ }^{1}$ Programa de Pós-Graduação em Geografia, Universidade Federal de Santa Catarina (UFSC), Florianópolis, SC, Brasil. \\ ${ }^{2}$ Fundação Estadual de Proteção Ambiental Henrique Luís Roessler (FEPAM), Porto Alegre, RS, Brasil. \\ ${ }^{3}$ Universidade Federal do Rio Grande do Sul (UFRGS), Porto Alegre, RS, Brasil. \\ *E-mail de contato: lauradp53@gmail.com
}

Artigo recebido em 28 de agosto de 2017, versão final aceita em 26 de novembro de 2017.

RESUMO: $\quad$ As Áreas de Proteção Ambiental (APAs) são Unidades de Conservação (UCs) de uso sustentável constituídas por terras públicas e privadas, onde diversos usos são permitidos. Entende-se que sua efetividade de gestão depende de uma melhor compreensão do contexto social em que estão inseridas. Além disso, é um dos principais instrumentos para a conservação no país e a categoria mais representativa do território brasileiro. Atualmente, existem dezenas de metodologias para Avaliação da Efetividade de Gestão, entretanto, ocorrem limitações para o uso dos métodos já conhecidos aplicados às APAs, pois é uma UC particular do Sistema Nacional de Unidades de Conservação e sem correspondência direta com as categorias da International Union for Conservation of Nature. O objetivo deste trabalho, portanto, é propor um método adequado de avaliação de efetividade de gestão de APAs devido as suas características únicas. Elaborou-se um método de Avaliação da Efetividade de Gestão de APAs, adaptando a metodologia Rapid Assessment and Prioritization of Protected Area Management (RAPPAM) à categoria APA com a colaboração de outras 11 metodologias. Como o RAPPAM é projetado para se fazer a avaliação de um grupo de unidades de conservação e avaliação de UCs florestais, a metodologia teve que ser adaptada para se adequar tanto para ser aplicada a apenas uma área como para APAs com territórios marinhos e continentais. O método foi testado aplicando-se o questionário para a equipe da APA da Baleia Franca (APABF), localizada no litoral sul de Santa Catarina. Observou-se que a APABF, por meio do método utilizado, possui uma efetividade de gestão de 49,4\%; assim, a efetividade de gestão dela é minimamente satisfatória. Este dado indica que a APABF não está cumprindo com os objetivos de conservação e se encontra altamente vulnerável. Concluiu-se que o método proposto atendeu a expectativa de avaliação de eficiência de gestão da APABF.

Palavras-chave: gestão; conservação; efetividade de gestão; área de proteção ambiental. 
ABSTRACT: The Environmental Protection Areas (APAs) categories are Conservation Units (CUs) with sustainable use of natural resources on private and public lands. It is well known that their management effectiveness depends on a better understanding of the social framework in which they are established. Besides, it is one of the principal tools for the conservation in Brazil and the most representative category in terms of area covering. Nowadays, there are several methodologies for evaluating and assessing the Management Effectiveness; however, there are limitations to the use of these methods applied to APAs, since it is a specific UC of the National System of Conservation Units and does not correspond directly with the categories of the International Union for Conservation of Nature. Thus, this work focuses on developing a suitable method to evaluating the management effectiveness of APAs. In this way, the Rapid Assessment and Prioritization of Protected Area Management (RAPPAM) with another 11 methodologies were adapted. As the RAPPAM methodology is designed to evaluate forest CUs, adaptive modifications were made to suit both a single area and APAs with marine and continental areas. The new methodology was tested by applying the questionnaire to a team from the APA of the Baleia Franca (APABF), located on the southern coast of Santa Catarina state. The results show that APABF has a management effectiveness of $49.4 \%$, therefore, minimally satisfactory. This indicates that the APABF is not reaching the conservation goals and is highly vulnerable. It was concluded that the proposed method here met the expectation of APABF management efficiency evaluation.

Keywords: management; conservation; management effectiveness; environmental protection area.

\section{Introdução}

O Sistema Nacional de Unidades de Conservação (SNUC) estabelece unidades de conservação (UCs) em duas categorias: de proteção integral e de uso sustentável, como é o caso das Áreas Proteção Ambiental (APAs), Reservas Extrativistas, Floresta Nacional, Área de Relevante Interesse Ecológico, Reserva de Fauna, Reserva de Desenvolvimento Sustentável e Reserva Particular do Patrimônio Natural. Para as unidades de uso sustentável, são permitidos o uso direto ${ }^{1}$ dos recursos naturais e a ocupação humana no seu interior. O objetivo desse grupo de UCs é "ordenar ações de caráter não sustentável, sobretudo as que têm impacto sobre o meio ambiente" (Guerra \& Coelho, 2009), restringir e regular usos e efeitos nocivos ao meio ambiente, provenientes de determinada atividade. Dessa forma, ao contrário das unidades de proteção integral, onde o uso do solo é extremamente restrito e nem a ocupação por populações tradicionais é permitida, as unidades de uso sustentável devem buscar uma interação maior entre sociedade e natureza.

Em 2010, uma reedição do estudo realizado pelo MMA identificou áreas prioritárias para conservação, uso sustentável e repartição de benefícios da biodiversidade brasileira das zonas costeira e marinha, e classificou mais da metade delas como áreas de importância biológica extremamente alta (MMA, 2010). A recomendação deste trabalho foi criar unidades de conservação nessas áreas. Dessa forma, perante a perda de habitats e o estágio de degradação dos ecossistemas costeiros, as unidades de conservação são vistas como o principal instrumento de conservação da biodiversidade (Cabral \& Souza 2005; Dourojeanni \& Pádua, 2007; Guimarães \& Pellin, 2015; Pureza et al., 2015). Estudos ainda afirmam que, no futuro, os remanescentes

\footnotetext{
${ }^{1}$ Uso direto é aquele que envolve coleta e uso, comercial ou não, dos recursos naturais (Brasil, 2000). Uso indireto é aquele que não envolve consumo, coleta, dano ou destruição dos recursos naturais (Brasil, 2000).
} 
de ambientes preservados pertencerão apenas às unidades de conservação (Orth \& Debetir, 2007; Guerra \& Coelho, 2009). No entanto, apesar de as áreas protegidas terem grande importância para a conservação da biodiversidade, além de respaldo jurídico e científico quanto a sua preservação, também enfrentam inúmeras dificuldades de implementação e gestão. Em particular, as unidades de conservação de uso sustentável, incluindo as Áreas de Proteção Ambiental, enfrentam dificuldades ainda maiores, em decorrência da presença humana no interior da unidade.

As APAs são UCs do grupo de uso sustentável, em geral extensas, constituídas por terras públicas e privadas, onde diversos usos são permitidos (MMA, 2010). De acordo com o Ministério do Meio Ambiente (MMA, 2010), é exatamente por conta dessas características que a efetividade da conservação proporcionada por uma APA depende da qualidade da gestão realizada e da implementação de um plano de manejo eficaz, que incorpore um zoneamento adequado aos usos existentes. Entende-se, também, que a grande extensão dessas unidades exige conselhos de gestão representativos de todos os municípios abrangidos, bem como dos diferentes tipos de usos presentes em seu interior, para estabelecer de forma negociada compromissos com a conservação (MMA, 2010). A efetividade da gestão dessas áreas, portanto, está sujeita a uma maior compreensão do contexto social no qual estão inseridas.

No entanto, ainda que o uso sustentável seja objetivo da APA, ele apresenta limitações como ferramenta básica para a conservação da biodiversidade (Bensusan, 2006). Decorre que as APAs, como categoria do SNUC, estão cercadas por uma série de questionamentos, derivados, principalmente, dos seus objetivos e da sua eficiência na conservação da biodiversidade, sendo que o principal deles é a própria permissão de uso direto dentro da UC. Mesmo diante de tantas discussões acerca de sua eficiência em promover a conservação da biodiversidade, a APA é a categoria espacialmente mais representativa no território brasileiro, conformam cerca de $30 \%$ das áreas cobertas por UCs no país (MMA, 2017). Compreende-se, portanto, que são necessários estudos que busquem a consolidação das APAs e que contribuam na promoção de meios de avaliação da efetividade gestão das APAs adequados às características particulares dessa categoria dentro do SNUC, com o fim de apoiar a gestão das mesmas.

A partir do exposto, damos continuidade ao texto, que se apresenta dividido em três partes. Primeiro, iniciaremos com algumas reflexões sobre as APAs, no que tange a sua trajetória dentro da legislação brasileira, à representatividade dentro do SNUC e aos debates recorrentes a essa categoria. Em seguida, introduzimos as discussões sobre a avaliação de efetividade dos instrumentos de gestão de áreas protegidas e a origem dos mesmos. Por último, apresentamos a metodologia elaborada nesse trabalho e os resultados da sua aplicação-piloto na APA da Baleia Franca.

\section{Areas de Proteção Ambiental: trajetória e debates}

\subsection{Trajetória da categoria APA na legislação brasileira}

As APAs foram criadas originalmente pela Lei 6.902, de 27 de abril de 1981. De acordo com essa Lei, a categoria APA possui o objetivo de assegurar o bem-estar das populações humanas e conservar ou 
melhorar as condições ecológicas locais. Limitava-se ou proibia as seguintes atividades: a implantação e o funcionamento de indústrias potencialmente poluidoras, que pudessem afetar nascentes de água; obras de terraplenagem e abertura de canais, quando essas afetassem as condições ecológicas locais; atividades que provocassem uma acelerada erosão das terras e/ou um acentuado assoreamento dos corpos hídricos; e o exercício de atividades que provocassem a extinção na área protegida de espécies raras da biota regional (Brasil, 1981). A Lei previa ainda que o não cumprimento das normas disciplinadoras implicaria aos infratores o embargo das iniciativas irregulares, obrigando a reposição e a reconstituição, além da aplicação de multas.

Em 2000, com a instituição do SNUC, a APA foi definida como uma área em geral extensa, com certo grau de ocupação humana, composta por atributos abióticos, bióticos, estéticos ou culturais importantes para a qualidade de vida e o bem-estar humano. Tem como "objetivos básicos proteger a diversidade biológica, disciplinar o processo de ocupação e assegurar a sustentabilidade do uso dos recursos naturais" (Brasil, 2000). Ela pode ser formada por áreas públicas ou privadas e podem ser estabelecidas normas e restrições para a utilização de uma propriedade privada localizada na UC. As APAs, dessa forma, teriam representado uma mudança de paradigma na conservação da biodiversidade, com a inclusão nos debates dos temas econômicos, sociais e culturais, admitindo que as populações humanas integrem este contexto (Bensusan, 2006; Souza, 2013). Historicamente, no mundo e no Brasil, somente a partir da década de
1980 foram criadas áreas protegidas de uso direto dos recursos naturais.

Em comparação com as outras categorias, a APA apresenta uma série de flexibilidades no que diz respeito a sua gestão e ao seu uso:

- A pesquisa científica e a visitação pública nas áreas sob domínio público serão estabelecidas pelo órgão gestor da unidade. Nas áreas de propriedade privada, o proprietário tem o direito de regular estes usos (Brasil, 2002);

- O conselho tem funções indeterminadas, podendo ser consultivos ou deliberativos ${ }^{2}$;

- Juntamente com as Florestas Nacionais, as Reservas Extrativistas e as Reservas do Desenvolvimento Sustentável, o SNUC abre exceções para a presença de animais e plantas não autóctones;

- Tal como as RPPNs, nas APAs não é necessária autorização para realizar a exploração comercial de produtos, subprodutos, serviços obtidos ou desenvolvidos a partir dos recursos naturais biológicos, cênicos, culturais ou da exploração de imagem da UC;

- Exige-se o licenciamento de empreendimento de significativo impacto ambiental dentro da UC ou de impacto direto nela;

- Não se exigem zonas de amortecimento;

- Tal como para as RPPNs, não se exige o licenciamento de empreendimento de significativo impacto ambiental por meio de Estudo de Impacto Ambiental e respectivo Relatório de Impacto Ambiental (EIA/RIMA), localizados numa faixa de três mil metros a partir do limite da UC;

\footnotetext{
${ }^{2}$ No SNUC, o caráter dos conselhos gestores das APAs não está definido. Uma definição foi dada com o Parecer nº 005/CONJUR/MMA/2003,
} da Consultoria Jurídica do Ministério do Meio Ambiente, que determinou como consultivos os conselhos de APAs (Ribeiro et al., 2010). 
- As UCs de proteção integral são as únicas beneficiárias diretas dos recursos provenientes de compensação ambiental de empreendimentos de significativo impacto ambiental. Quando uma unidade de Uso Sustentável é diretamente afetada, os recursos podem somente custear atividades do plano de manejo, de educação ambiental e de pesquisas científicas que visem ao manejo da área. No entanto, não são as únicas beneficiárias, dividindo os recursos com outras unidades.

Destaca-se que as APAs ainda não possuem regulamento. A Resolução CONAMA n ${ }^{\circ} 10$, de 14 de dezembro de 1988, que dispõe sobre a regulamentação das APAs, foi revogada. A Resolução $\mathrm{n}^{\circ} 428$, de 2010, que dispõe basicamente sobre licenciamento ambiental em UCs, nada disserta de forma específica sobre APAs. Compreende-se com isso que houve uma perda de marco legal para as APAs. A citada Resolução definia, por exemplo, que as APAs deveriam possuir um Zoneamento Ecológico-Econômico, atualmente importante instrumento da Política Nacional do Meio Ambiente e do Plano Nacional de Gerenciamento Costeiro.

\subsection{Debates sobre as APAs}

De acordo com o Cadastro Nacional de Unidades de Conservação (MMA, 2017), atualmente existem 650 UCs de proteção integral, abrangendo $546.292 \mathrm{~km}^{2}$, isto é, 4,48\% do país. As UCs de uso sustentável somam 1.421 e cobrem outros $1.039 .486 \mathrm{~km}^{2}$ ou $8,5 \%$ do território brasileiro (continental e marinho) ${ }^{3}$. Dessa forma, observa-se que, no Brasil, a maior parte da superfície coberta por UCs é de uso direto. De todas as categorias de uso direto, a APA, quase desconhecida em outros países, é a que tem sido dominante na expansão das UCs brasileiras (Dourojeanni \& Pádua, 2007). Portanto, a categoria mais representativa no território brasileiro é a APA, a qual constitui cerca de $30 \%$ das áreas cobertas por UCs no país (MMA, 2017). Em números, são 305 APAs, que somam 469.506 $\mathrm{km}^{2}$ ou aproximadamente $3,8 \%$ da área do sistema de UCs (ver Figuras 1 e 2).

Segundo Cabral \& Souza (2005), compreende-se que existem três formatos de Unidades de Conservação (UCs) no Brasil: (i) as UCs que não permitem, sob nenhum aspecto, a presença humana, excetuando-se a visitação pública; (ii) as UCs que permitem a presença humana somente sob a forma de populações tradicionais e povos indígenas; (iii) as que permitem a presença do homem e o desenvolvimento de atividades de uso direto dos recursos naturais no interior das UCs. Estas últimas são

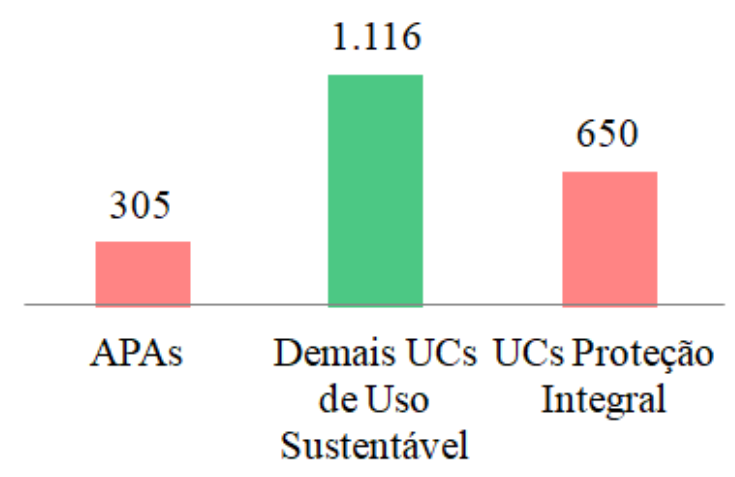

FIGURA 1 - Número de UCs no Brasil. FONTE: MMA (2017).

\footnotetext{
${ }^{3}$ Os dados foram calculados com base no território continental $\left(8.516 .000 \mathrm{~km}^{2}\right)$ mais a Zona Econômica exclusiva $\left(3.660 .955 \mathrm{~km}{ }^{2}\right)$, somando uma área total de $12.176 .955 \mathrm{~km}^{2}$.
} 

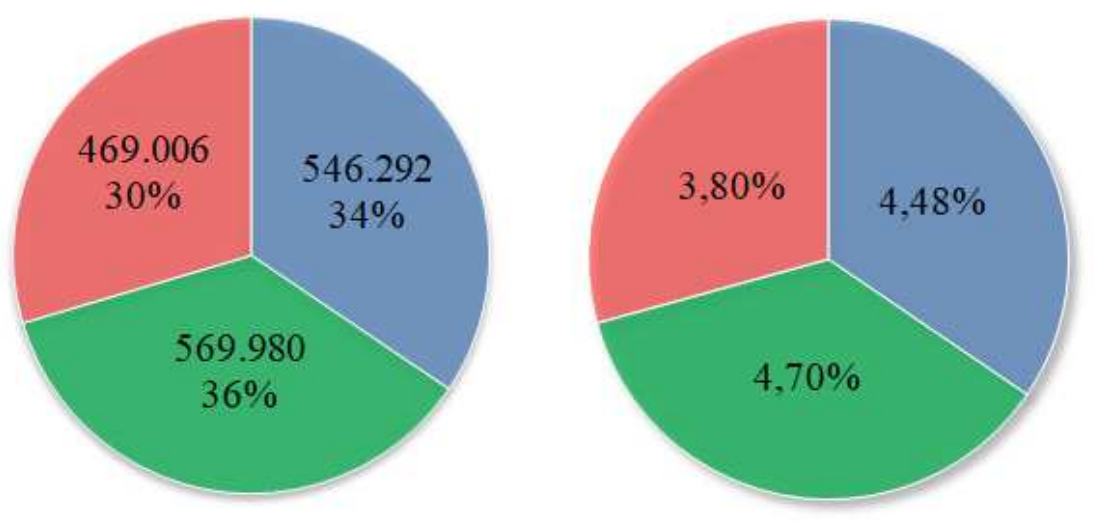

UCs de Proteção Integral

- Demais UCs de Uso Sustentável

APAs

FIGURA 2 - Áreas da UCs no Brasil (km2); percentagem do território brasileiro coberto por UCs.

FONTE: MMA (2017).

controladas pelo Estado, como é o caso das Áreas de Proteção Ambiental (APAs).

Entretanto, as APAs nem sempre são vistas como UCs eficientes para garantir a conservação da biodiversidade, fazendo com que exista, para este propósito, maior preferência pelas UCs de proteção integral (Cabral \& Souza, 2005; Bensusan, 2006; Dourojeanni \& Pádua, 2007; Guerra \& Coelho, 2009; (Pureza et al., 2015). Segundo Rodrigues (2005), uma APA onde qualquer atividade humana é possível "dentro da lei" não oferece nenhuma proteção à biodiversidade, embora seja melhor que uma área sem proteção. Já de acordo com Bensusan (2006), as APAs, enquanto instrumento de conservação no SNUC, refletem uma tentativa de aliança entre o princípio da conservação do meio ambiente e o desenvolvimento econômico.

Nesse caso, a APA é uma categoria bastante particular no SNUC, na medida em que permite a presença humana em suas terras, sendo estas predominantemente privadas, "fazendo com que, exceção feita ao zoneamento (que raramente é respeitado), seja pouco diferente de qualquer outra parte do território nacional, onde se respeita a legislação orgânica em vigor" (Dourojeanni \& Pádua, 2007). Para Pureza et al. (2015), as APAs já são criadas envoltas por contestações em função das suas aplicabilidade e viabilidade no país. Questiona-se, por exemplo, se a categoria não se tornou um mero instrumento utilizado por governantes para a realização de propaganda política e disfarçar os números de espaços efetivamente protegidos no Brasil (Pureza et al., 2015). Nas críticas relacionadas às APAs, também se insere a questão sobre o crescimento exagerado do número e da área de UCs de uso direto e a falsa sensação de que o território está ambientalmente protegido. O sistema de unidades de conservação do país possui $66 \%$ do total da área de suas UCs composto por unidades de uso sustentável. Essa situação, no entendimento de muitos pesquisadores, gera o falso entendimento de que a biodiversidade está protegida e que existem áreas protegidas em demasia (Cabral \& Souza, 2005). Dourojeanni \& Pádua (2007) também comentam sobre este assunto 
e afirmam que o problema não é a existência de categorias de uso direto: o problema é o abuso.

Outro ponto a ser considerado é a dificuldade de gestão desse tipo de UC. Para Dourojeanni \& Pádua (2007), a margem de manobra dos que manejam UCs de uso direto se vê seriamente limitada pelos direitos de propriedade e/ou de uso. Dessa maneira, as dificuldades de conciliação de interesses entre usuários, proprietários e gestores prejudicam a eficiência. Segundo Cabral \& Souza (2005), a dificuldade na gestão pode ser o resultado de decretos de criação pouco específicos e com objetivos gerais ou restritivos, prejudicando a identidade desse tipo de UC. O SNUC confere autonomia administrativa às UCs, porém, o município também possui autonomia administrativa. Essa situação sugere que os agentes administrativos, tanto da prefeitura como da gestão da UC, "comunguem da mesma ideia de preservação de determinada área, com objetivo em comum e procedimentos de manutenção acordados" (Cabral \& Souza, 2005). Contudo, esta é uma situação ideal, na realidade essa situação é de conflito e causa inércia no processo de gestão da UC.

\section{Avaliação de efetividade de gestão de áreas protegidas}

\subsection{Considerações sobre o conceito de gestão}

Observa-se uma grande variedade de termos para expressar as atividades realizadas em UCs. Manejo e gestão são alguns deles, os quais são usados muitas vezes como sinônimos (Faria, 2004; Gidsicki, 2012). Segundo Faria (2004), as unidades de conservação têm sofrido um acréscimo de desafios e problemas a enfrentar; em função disso, o conceito de manejo de UCs, emprestado do conceito de manejo de ecossistemas, evoluiu para dar conta da amplitude de ações que, cada vez mais, são desenvolvidas nelas.

Nesse sentido, o termo manejo é aplicado para designar uma série de atividades referentes às UCs e, conforme Faria \& Pires (2012), é o termo mais utilizado na América Latina, inclusive no Brasil. Sua origem vem das palavras inglesas management e manager, que correspondem a administração e a administrador, e seu significado no dicionário português é manusear, manipular com as mãos (Faria, 2004).

Uma das definições de manejo de áreas protegidas mais usadas é a do pesquisador costa-riquenho Miguel Cifuentes. Para este autor, o manejo integra um conjunto de ações no âmbito político, legal, administrativo, de pesquisa, de planejamento, de proteção, de coordenação, de promoção, de interpretação e de educação, entre outros, que dão como resultado o melhor aproveitamento e eficiência de uma área protegida no cumprimento de seus objetivos. Ele associou de forma direta manejo à eficiência/efetividade de conservação, entendendo que o manejo é a totalidade de ações (políticas, financeiras, ambientais, etc.) suficientes para se ter um bom nível de conservação. Nesse entendimento, a efetividade de manejo é alcançada quando o conjunto de ações baseadas na capacidade e aptidões de cada área protegida (manejo) permite cumprir satisfatoriamente o objetivo para o qual foi criada a área protegida (Cifuentes et al., 2000). Assim, a efetividade de manejo se dá quando a eficiência dele resulta em impacto positivo de conservação (Gidsicki, 2012). 
O SNUC define manejo de uma UC como todo e qualquer procedimento que vise assegurar a conservação da diversidade biológica dos ecossistemas (Brasil, 2000). Este seria um termo "guarda-chuva", o qual abrigaria todas as tendências filosóficas (Faria, 2004). No entanto, concordamos com Gidsicki (2012) quando afirma que o termo manejo possui o sentido de "a manipulação dos recursos naturais renováveis que são levados a cabo em determinados setores socioeconômicos ou tipos específicos de sistemas ambientais, como manejo da vegetação, manejo da fauna, manejo de solos". Dito isso, embora o conceito de manejo formulado por Cifuentes et al. (2000) seja abrangente, a palavra em português possui um cunho limitado para representar toda a complexidade do processo que ela tende a descrever.

A concepção de gestão, por sua vez emprestada da administração para a área ambiental, passou a ser amplamente utilizada. No dicionário português, seu significado é o ato de gerir, administração, gerência. Nessa definição, a gerência cuidaria de "supervisionar e coordenar todos os procedimentos relacionados a um produto específico" (Faria, 2004). Nesse caso, é preciso atuar sobre todos os elementos que integram as UCs para alcançar os objetivos de conservação. Faria (2004) define gestão de unidades de conservação como "a equilibrada coordenação dos componentes técnicos e operacionais (recursos humanos, materiais, financeiros) e os diversos atores sociais que incidem sobre o desenvolvimento da área", proporcionando a eficácia desejada, com o alcance dos objetivos da UC e conservação de seus ecossistemas.

\subsection{Métodos de Avaliação de Efetividade de Gestão}

Management Effectiveness Evaluation (MEE), termo que passou a ser comumente utilizado nas metodologias e nos relatórios internacionais, e que para o português foi traduzido como "Avaliação da Efetividade de Gestão", reflete três temas principais: (i) questões de delimitação da área, relativas a unidades individuais ou sistemas de áreas protegidas; (ii) adequação e apropriação nos sistemas de gestão e processos de gestão e; (iii) cumprimento dos objetivos da área protegida, incluindo valores de conservação (Hockings et al., 2006).

As discussões sobre a necessidade de se fazer uma avaliação na gestão das áreas protegidas como instrumento para se melhorar a efetividade na conservação da natureza foram introduzidas no III Congresso Mundial de Parques, realizado em Bali, em 1982. Dez anos depois, no IV Congresso Mundial de Parques, realizado em Caracas, essa mesma discussão voltou à tona e definiu-se como prioridade o desenvolvimento de métodos que pudessem avaliar a efetividade de gestão das áreas protegidas. Para fomentar estas ações, foi criado o Grupo de Trabalho da Comissão Mundial de Áreas Protegidas (WDPA/ IUCN) em 1995. O grupo elaborou uma proposta, publicada em 2000, de ciclo de gestão e avaliação, a partir do qual se propôs um quadro de elementos (Framework IUNC-WDPA) para se avaliar a efetividade na gestão de unidades de conservação. Essas diretrizes de avaliação lançadas pela IUCN influenciaram de forma determinante a elaboração e a aplicação de várias metodologias pelo mundo.

Uma segunda edição do quadro IUCN-WDPA (Tabela 1) foi lançada em 2006. De acordo com Leverington et al. (2008a), o quadro não é, em si, uma metodologia específica para avaliar a eficácia da gestão, mas um quadro de desenvolvimento de sistemas de avaliação e orientação para a prática da 
avaliação. Ele é baseado na ideia de que a gestão de áreas protegidas segue um processo com seis fases distintas ou elementos (Leverington et al., 2008a).

Dessa forma, destaca-se que o quadro publicado em 2000 pela UICN-WDPA foi consagrado com um "marco de referência para a avaliação da gestão de áreas protegidas" (Leverington et al., 2008a). Em 2004, na $7^{\mathrm{a}}$ Conferência das Partes da Convenção sobre Diversidade Biológica, os 187 países signatários e um bloco regional se comprometeram em desenvolver metodologias de avaliação e aplicar em $30 \%$ de suas áreas protegidas.

Já em 2008, a WDPA-IUCN publica o Estudo Global de Avaliações de Efetividade de Gestão em Áreas Protegidas e, juntamente com este, lança um relatório complementar com uma visão geral das abordagens e das metodologias desenvolvidas pelo mundo. No ano de 2010, a segunda edição do Estudo é publicada e, dessa vez, o relatório complementar trata apenas das avaliações de efetividade de áreas protegidas na Europa. O primeiro Estudo Global registrou mais de 6.300 avaliações de eficácia da gestão em 100 países e 42 métodos diferentes, o que abrangeu somente $6 \%$ do total de mais de 100.000 áreas protegidas cadastradas pelo Banco de Dados Mundial de Áreas Protegidas (WDPA). A segunda edição do Estudo registrou mais de 60 metodologias diferentes e com 9.000 avaliações em 140 países.

Observa-se que o Framework UICN-WDPA se estrutura em seis elementos: contexto, planejamento, entradas, processos, saídas e resultados. Comparando com o ciclo de gestão, o contexto refere-se ao estado atual da gestão e nesta etapa devem ser identificados as ameaças, a vulnerabilidade e o ambiente político. Já o planejamento refere-se aos objetivos, ao amparo político-institucional e à delimitação da área protegida. As entradas ou insumos são os recursos humanos, financeiros e a

TABELA 1 - Framework IUCN-WDPA para avaliar a eficácia da gestão de áreas protegidas

\begin{tabular}{|c|c|c|c|c|c|c|}
\hline & \multicolumn{2}{|c|}{ Projeto } & \multicolumn{2}{|c|}{ Apropriação/Adequação } & \multicolumn{2}{|c|}{ Entrega } \\
\hline $\begin{array}{l}\text { do ciclo de } \\
\text { gestão }\end{array}$ & Contexto & Planejamento & Entradas & Processos & Saídas & Resultados \\
\hline $\begin{array}{c}\text { Foco de } \\
\text { avaliação }\end{array}$ & $\begin{array}{l}\text { Avaliação da } \\
\text { importância, ame- } \\
\text { aças e políticas } \\
\text { ambientais }\end{array}$ & $\begin{array}{l}\text { Avaliação da } \\
\text { área protegida, } \\
\text { projeto e plane- } \\
\text { jamento }\end{array}$ & $\begin{array}{l}\text { Avaliação dos } \\
\text { recursos necessá- } \\
\text { rios à execução } \\
\text { do gerenciamento }\end{array}$ & $\begin{array}{l}\text { Avaliação da for- } \\
\text { ma como a gestão } \\
\text { é conduzida }\end{array}$ & $\begin{array}{l}\text { Avaliação da im- } \\
\text { plementação dos } \\
\text { programas e ações } \\
\text { da gestão; entrega } \\
\text { de produtos e } \\
\text { serviços }\end{array}$ & $\begin{array}{l}\text { Avalia- } \\
\text { ção dos } \\
\text { resultados } \\
\text { e o grau do } \\
\text { alcance dos } \\
\text { objetivos }\end{array}$ \\
\hline $\begin{array}{l}\text { Critérios de } \\
\text { avaliação }\end{array}$ & $\begin{array}{c}\text { Significância, } \\
\text { valores, ameaças, } \\
\text { vulnerabilidade } \\
\text { das partes interes- } \\
\text { sadas, contexto } \\
\text { nacional }\end{array}$ & $\begin{array}{c}\text { Legislação, po- } \\
\text { lítica e desenho } \\
\text { da área protegi- } \\
\text { da, desenho do } \\
\text { sistema de áreas } \\
\text { protegidas, } \\
\text { gestão e plane- } \\
\text { jamento }\end{array}$ & $\begin{array}{l}\text { Recursos dis- } \\
\text { poníveis para a } \\
\text { área protegida e } \\
\text { recursos disponí- } \\
\text { veis para o órgão } \\
\text { gestor }\end{array}$ & $\begin{array}{l}\text { Adequação do } \\
\text { processo de ges- } \\
\text { tão e a extensão } \\
\text { que eles são } \\
\text { estabelecidos ou } \\
\text { aceitos ao serem } \\
\text { implementados }\end{array}$ & $\begin{array}{c}\text { Os resultados } \\
\text { das ações de } \\
\text { gestão; serviços e } \\
\text { produtos }\end{array}$ & $\begin{array}{l}\text { Impactos: } \\
\text { efeitos da } \\
\text { gestão em } \\
\text { relação aos } \\
\text { objetivos }\end{array}$ \\
\hline
\end{tabular}

FONTE: Hockings et al. (2006). 
infraestrutura da UC. Os processos são as ações, os projetos e os planos que estão sendo elaborados ou implementados para que se atinjam os objetivos da UC. As saídas configuram as ações, os projetos e os planos que já foram realizados para que se atinjam os objetivos da UC. Os resultados, por sua vez, procuram avaliar se algumas ações inerentes a uma boa gestão, como monitoramento, mitigação de danos e planejamento, por exemplo, foram realizadas.

\section{Método para avaliar a efetividade de gestão das Áreas de Proteção Ambiental: uma adaptação a partir do RAPPAM}

O ICMBio, em parceria com o WWF-Brasil, desenvolveu um ciclo de avaliação 2006-2010 para as unidades de conservação federais (ICMBio, 2012). O método utilizado foi uma adaptação do Rapid Assessment and Prioritization of Protected Area Management - RAPPAM (Ervin, 2003). O RAPPAM é baseado no Framework IUCN-WDPA.

O RAPPAM é uma metodologia de avaliação da efetividade de gestão que foi desenvolvida pela WWF, entre 1999 e 2002. Ela já foi utilizada em 53 países e em mais de 1.600 áreas protegidas na Europa, Ásia, África, América Latina e Caribe (Leverington et al., 2010). O método tem como objetivo observar e identificar o grau de ameaças enfrentadas pelo sistema de unidades de um país ou região, permitindo uma visão do universo de problemas enfrentados pelas UCs, bem como as formas como elas estão funcionando e realizando a gestão. $\mathrm{O}$ foco converge para a avaliação dessas tendências com o intuito de corrigir e melhorar a eficiência na gestão.
A Metodologia RAPPAM tem como finalidades: a) identificar os pontos fortes e fracos do manejo; b) analisar o escopo, a severidade, a predominância e a distribuição das diversas ameaças e pressões; c) identificar áreas de alta importância ecológica e social e sua vulnerabilidade; d) indicar a urgência e a prioridade de conservação de unidades de conservação individuais; e) ajudar no desenvolvimento e na priorização de intervenções políticas adequadas e dos passos seguintes de acompanhamento para melhorar a efetividade de manejo de unidades de conservação. (Leverington et al., 2008a).

A escolha dessa metodologia como base para o questionário se deu principalmente em função dos seguintes motivos: a) fácil adaptação; b) tempo de aplicação adequado à pesquisa em questão; c) ser o método utilizado pelo ICMBio para avaliar 246 unidades em 2006 e 292 unidades em 2010 no ciclo de avaliação 2006-2010 (ICMBio, 2012), incluindo as APAs; e d) estar de acordo com o Framework IUCN-WDPA.

No entanto, o RAPPAM é direcionado às categorias I a IV da IUCN, mesmo que possa ser aplicado em categorias V e IV, correspondentes, respectivamente, às APAs e às Reservas Extrativistas. Nesse caso, a metodologia indica que, se for aplicado nessas categorias, os indicadores de práticas de manejo e foco nas comunidades devem ser mais abrangentes. Outro ponto é que se indica a aplicação do RAPPAM para unidades de conservação florestais, não correspondendo a áreas protegidas com uso direto dos recursos naturais e outras variações de ambiente. Além disso, a metodologia RAPPAM não foi desenvolvida para ser aplicada em apenas uma área protegida; para isso, também são indicadas adaptações. 
Como o alvo de pesquisa é apenas uma unidade, sendo uma APA, a metodologia RAPPAM teve que ser adaptada para se adequar, tanto para ser aplicada a apenas uma área como para uma unidade de conservação de uso sustentável, com territórios marinhos e/ou continentais. Dessa forma, elaborou-se uma metodologia para avaliar APAs a partir de uma adaptação do método RAPPAM, com

TABELA 2 - Metodologias de $M E E$ que colaboraram com a adaptação do RAPPAM

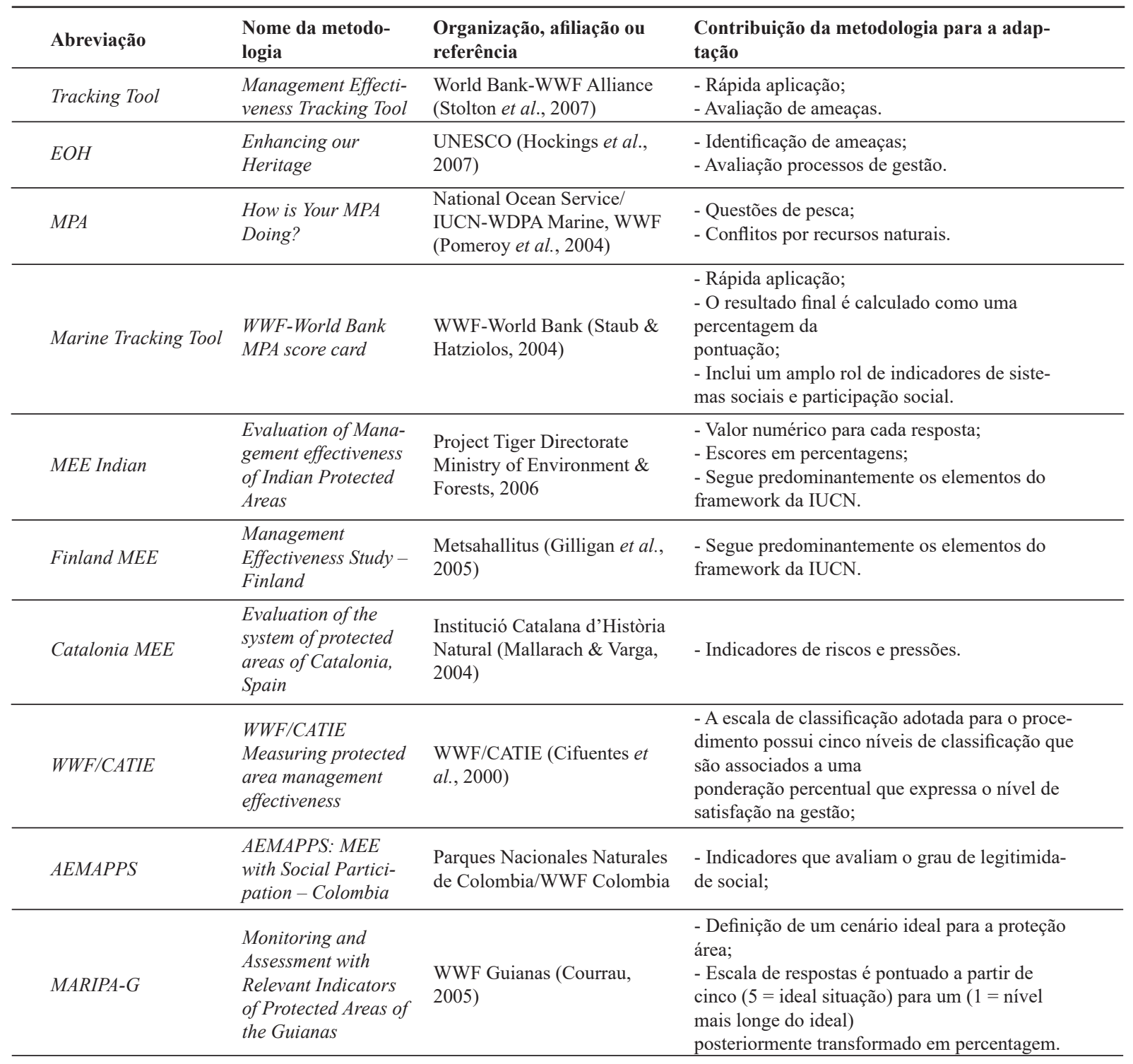


colaboração de outras 11 metodologias (Tabela 2). Estas 11 metodologias foram pesquisadas por meio do Relatório Complementar do Estudo Global (Leverington et al., 2008b) sobre avaliação da efetividade de gestão em áreas protegidas, já mencionado aqui. Entre as 42 metodologias descritas nesse relatório, as selecionadas para apoiar a adaptação do RAPPAM foram escolhidas com base nas seguintes necessidades/características: a) rápida aplicação e avaliação; b) destinadas à avaliação de apenas uma área protegida; c) baseadas no framework da IUCN; d) possibilidades de respostas fechadas; e e) possuir indicadores no contexto do uso direto e sustentável do território da área protegida. Assim, das 42 metodologias pesquisadas, 11 delas possuem pelos menos uma dessas características. O intuito foi embasar os indicadores conservados do RAP$P A M$ original para a adaptação e ter sugestões para: a) rol de perguntas e indicadores; b) possibilidades de respostas; c) forma de aplicação e avaliação do questionário; d) escala de escores e cálculo do resultado final; e) inclusão de indicadores que considerassem as questões sociais e não exclusivamente a análise biológica ou de cunho preservacionista; e f) definição dos cenários de efetividade de gestão.

A primeira adequação realizada foi excluir o módulo "perfil" (Tabela 3), depois foi dividir o elemento contexto em dois, pois a importância biológica e a importância socioeconômica não fo- ram consideradas indicadores que permitam medir o grau de efetividade de gestão e de conservação. Considerar, por exemplo, que área protegida possui um nível relativamente alto de endemismo ou se as comunidades locais dependem de recursos naturais da área protegida para a sua subsistência não medem uma "eficiência" ou um "cumprimento de objetivos". Pondera-se que uma área protegida pode possuir uma ampla biodiversidade em seu território, mas apenas sua criação legal não garante a conser-

TABELA 3 - Estrutura do questionário RAPPAM original (ICMBio, 2012)

\begin{tabular}{ll}
\hline Elementos & Módulo temático \\
\hline 1. Perfil & 2. Pressões e ameaças \\
3. Importância biológica & 4. Importância socioeconômica \\
& 5. Vulnerabilidade \\
\hline Planejamento & 6. Objetivos \\
& 7. Amparo legal \\
& 8. Desenho e planejamento da área \\
\hline 9. Recursos humanos & 10. Comunicação e informação \\
1nsumos & 11. Infraestrutura \\
& 13. Recursos financeiros \\
\hline Processos & 14. Tomada de decisão \\
& 15. Pesquisa, avaliação e monitoramento \\
\hline Resultados & 16. Resultados \\
\hline
\end{tabular}


vação dessa biodiversidade, ou seja, ela precisa ser efetivada com uma gestão. Mesmo assim, estes dois elementos não foram excluídos do questionário, pois seus resultados podem ser interpolados com os resultados dos outros módulos. Dessa forma, o contexto "um" não irá integrar a compilação quantitativa da avaliação.

O módulo 2, "Pressão e ameaças", foi inteiramente reestruturado. Primeiramente, foi substituído por impactos no meio biofísico - severidade e abrangência. As possíveis respostas foram alteradas para "muito alta" a "nula". Para os impactos no meio biofísico também foi elaborada uma relação de impactos (passível de adaptação) a partir de uma lista padrão de atividades. São elas: a) extração de madeira; b) agricultura; c) silvicultura; d) pastagem; e) extração mineral; f) caça; g) turismo e recreação; h) aquicultura; i) maricultura; j) pescas continental e marinha; k) infraestrutura de transportes; 1) ocupações urbanas; m) drenagem de áreas unidas; o) poluição por efluentes industriais, domésticos e de mineração em mananciais fluviais, marinhos e subterrâneos; p) incêndios de causas naturais e antrópicos, q) invasão de espécies exóticas animais e vegetais. Foram incluídos também impactos de atividades específicas sobre alvo de conservação da área protegida. Nesse sentido, o objetivo foi relacionar a diversidade de atividades que podem ser desenvolvidas dentro de uma APA, embora seja sabido que os impactos e suas atividades relacionadas presentes em uma área protegida dependem das características específicas das mesmas.

Em relação às perguntas, excetuando os módulos 1 e 2 da metodologia original, oito delas (questões 18, 84, 85, 86, 97, 135, 146 e 147) foram adicionadas ao questionário integrando temáticas de âmbito social, valor turístico, monitoramento de atividades, educação ambiental e consciência ecológica da população. Cinco perguntas foram adaptadas para questões de projetos, planos e políticas coerentes com os objetivos, comunicação com os atores socais, modelo que otimiza a conservação da biodiversidade e estratégias para enfrentar ameaças, referentes às questões 79, 80, 93, 107 e 118. Outras quinze perguntas foram excluídas por não se adequarem à categoria APA e à análise de apenas uma UC. Estas alterações foram feitas a partir do questionário original do RAPPAM na versão aplicada nos ciclos 2005-06 e 2010 (ICMBio, 2012).

Deste modo, o questionário foi dividido em cinco elementos (Tabela 4): contexto, planejamento, insumos, processos e resultados, distribuídos em 16 módulos temáticos: Contexto 1 (Importância biológica e Importância socioeconômica); Contexto 2 (Impactos no meio biofísico [abrangência], Impactos no meio biofísico [severidade] e Vulnerabilidade); Planejamento (Objetivos, Amparo legal e Desenho e planejamento da área); Insumos (Recursos humanos, Comunicação e informação, Infraestrutura e Recursos financeiros); Processos (Planejamento e gestão, Processo de tomada de decisão e Pesquisa, avaliação e monitoramento); e Resultados (Resultados), com um total de 147 perguntas ${ }^{4}$.

Para a contagem dos resultados, um peso relativo foi atribuído a todos os indicadores, ou seja, o método considera que todos os aspectos avaliados têm o mesmo nível de importância

\footnotetext{
${ }^{4} \mathrm{O}$ questionário completo aplicado na APA da Baleia Franca está disponível para visualização no link: $<$ https://drive.google.com/file/d/0B_MNYCm3QdAjTjJFb1BMLUhvWU0/view?usp=sharing>.
} 
TABELA 4 - Estrutura do questionário RAPPAM adaptada

\begin{tabular}{|c|c|}
\hline Elementos & Módulo temático \\
\hline \multirow{2}{*}{ Contexto 1} & 1. Importância biológica \\
\hline & 2. Importância socioeconômica \\
\hline \multirow{3}{*}{ Contexto 2} & 3. Impactos no meio biofísico (ABRANGÊNCIA) \\
\hline & 4. Impactos no meio biofísico (SEVERIDADE) \\
\hline & 5. Vulnerabilidade \\
\hline \multirow{3}{*}{ Planejamento } & 6. Objetivos \\
\hline & 7. Amparo legal \\
\hline & 8. Desenho e planejamento da área \\
\hline \multirow{4}{*}{ Insumos } & 9. Recursos humanos \\
\hline & 10. Comunicação e informação \\
\hline & 11. Infraestrutura \\
\hline & 12. Recursos financeiros \\
\hline \multirow{3}{*}{ Processos } & 13. Planejamento e gestão \\
\hline & 14. Tomada de decisão \\
\hline & 15. Pesquisa, avaliação e monitoramento \\
\hline Resultados & 16. Resultados \\
\hline
\end{tabular}

FONTE: Autores.

para avaliar se a APA está cumprindo com seus objetivos de conservação e eficiência na gestão. Dentre os módulos que entraram na compilação quantitativa dos dados, os módulos 3 e 4 foram diferenciados dos demais. Estes abrangem duas informações diferentes: severidade e abrangência do impacto. As respostas possíveis foram: Muito Alta, Alta, Média, Baixa, Nula e Desconheço, para as quais foram designados os valores 1, 2, 3, 4, 5 e 0 , respectivamente. Para todos os outros módulos, as respostas possíveis foram: Sim; Predominantemente Sim; Médio; Predominantemente Não; Não; e Desconheço, para os quais foram atribuídos os respectivos valores: $5,4,3,2,1$ e 0 , exceto para o módulo 5, "Vulnerabilidade", para o qual os valores serão dados de forma invertida: para o "Sim", foi atribuído escore 1, para "Predominantemente Sim", o escore 2, para o "Médio", escore 3, para "Predominantemente Não", escore 4, para o "Não", escore 5 e 0 para "Desconheço". Explica-se: as afirmações dos módulos 6 a 16 indicam uma situação ideal de gestão, o nivelamento das respostas define o grau de satisfação dessa situação ideal, onde o "Não" corresponde à inexistência desse cenário. A vulnerabilidade questionada no módulo 5 expressa que, se a variável não existe, indica um favorecimento à gestão e a pontuação dada deve ser a máxima, no caso, 5 pontos. A opção de resposta "Desconheço" foi inserida em todas as perguntas e, para esta resposta, foi dada a pontuação " 0 ”. Assim, a pontuação adotada para o procedimento tem seis níveis de classificação (de 0 a 5), que serão associados a um percentual expressando uma situação, conforme a Tabela 5.

O percentual de igual ou inferior a $35 \%$ do ideal é considerado insatisfatório e indica que a gestão da área protegida não tem o mínimo de recursos para assegurar a sua gestão básica. Sua permanência em longo prazo está comprometida e, com tais condições, não é possível satisfazer os objetivos de conservação da área protegida. Valores entre $36-50 \%$ correspondem a "minimamente satisfatório", ou seja, a área protegida tem alguns recursos fundamentais para a gestão, mas minimamente

TABELA 5 - Sistema de pontuação usado no processo de avaliação

\begin{tabular}{cc}
\hline$\%$ & Classificação \\
\hline $0<35$ & Não satisfatório \\
$36-50$ & Minimamente satisfatório \\
$51-75$ & Moderadamente satisfatório \\
$76-90$ & Satisfatório \\
$91-100$ & Muito satisfatório \\
\hline
\end{tabular}

FONTE: Leverington et al. (2008b). 
aceitáveis. A área protegida é altamente vulnerável e seus objetivos de conservação não estão garantidos. Resultados entre $52-75 \%$ representam uma situação moderadamente satisfatória. Isto significa que a área possui requisitos mínimos para a sua gestão, mas ainda tem deficiências essenciais que comprometem uma gestão eficaz. O cumprimento dos objetivos é parcial. Entre 76-89\%, ou situação satisfatória, indica que as atividades administrativas estão adequadamente sendo atendidas e os objetivos de conservação estão garantidos, embora permitam melhorias. Valores entre $90-100 \%$ são considerados como uma situação muito satisfatória de conservação, ou seja, a área recebe todo o apoio necessário para a sua gestão e seus objetivos estão sendo totalmente atingidos (Leverington et al., 2008b).

Para a aplicação dessa metodologia, é necessário que o público-alvo tenha conhecimento de questões bastante específicas do território da APA e da gestão da mesma. Por isso, se não forem rea- lizadas oficinas de nivelamento com demais públicos-alvo, como o conselho da APA, o questionário só deve ser aplicado a gestores e profissionais que possuam o conhecimento adequado para prover dados suficientes e confiáveis (ICMBio, 2012). Como o objetivo da metodologia é essencialmente uma avaliação rápida do processo de gestão, não é indicada, embora possa ocorrer, a realização de oficinas. Nesse contexto, para o teste do método, aplicou-se o questionário para os seis analistas ambientais da APA Baleia Franca, como apresentaremos a seguir.

\subsection{Aplicação do método: efetividade de gestão da APA da Baleia Franca}

APA da Baleia Franca (APABF) é uma unidade de conservação federal de ambiente marinho-costeiro. Localizada no litoral centro-sul do Estado de Santa Catarina, possui uma área de 154.866,27 ha (Figura 3) e integra os municípios de Palhoça, Paulo

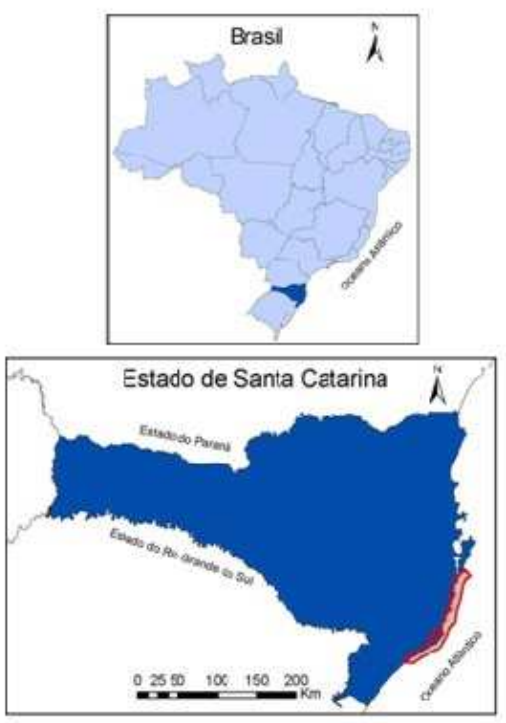

FIGURA 3 - Localização da APA da Baleia Franca.

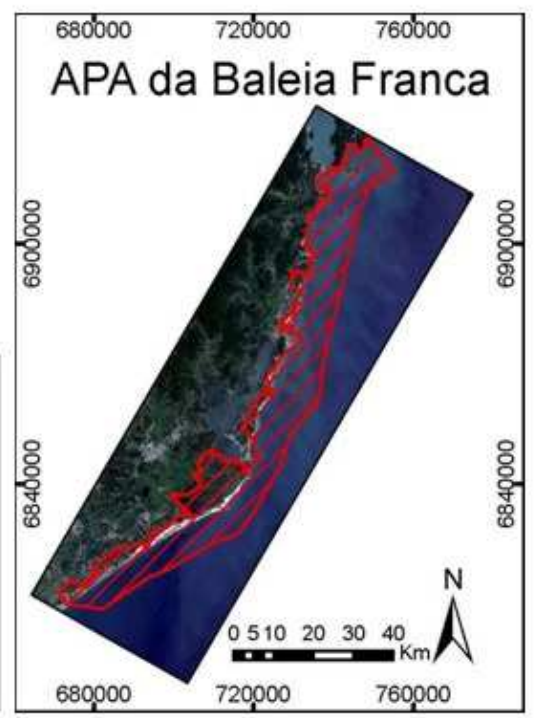

FONTE: Autores. 
Lopes, Garopaba, Imbituba, Tubarão, Florianópolis, Laguna, Jaguaruna e Balneário Rincão. Foi criada por meio de decreto em 14 de setembro de 2000. Tem aproximadamente $130 \mathrm{~km}$ de costa, sendo que $80 \%$ da área é marinha e o restante, terrestre.

A metodologia foi aplicada em fevereiro de 2016 para os seis analistas ambientais da APABF. Como o questionário é composto totalmente de questões objetivas, no próprio documento existem orientações sobre a situação ideal a ser considerada e o escore dado para cada resposta. Sendo assim, não foi necessário o acompanhamento dos entrevistados durante a aplicação dos questionários. Posteriormente, os dados foram tabulados e analisados com a ajuda do software Sphinx, direcionado para análise de pesquisas qualitativa e quantitativa feitas com questionários.

Os resultados do método indicaram que o módulo em que a gestão da APABF possui menos efetividade é "Pesquisa, Avaliação e Monitoramento", seguido pelos módulos "Vulnerabilidade" e "Impactos no Meio Biofísico" em nível de abrangência (Figura 4). Nota-se que, apesar de a unidade possuir alta importância biológica e socioeconômica, a sua vulnerabilidade é alta e a abrangência dos impactos também. O módulo "Tomada de Decisão" apresenta uma efetividade de $74,6 \%$, seguido do módulo "Objetivos", com 60,8\%, e "Recursos Humanos",

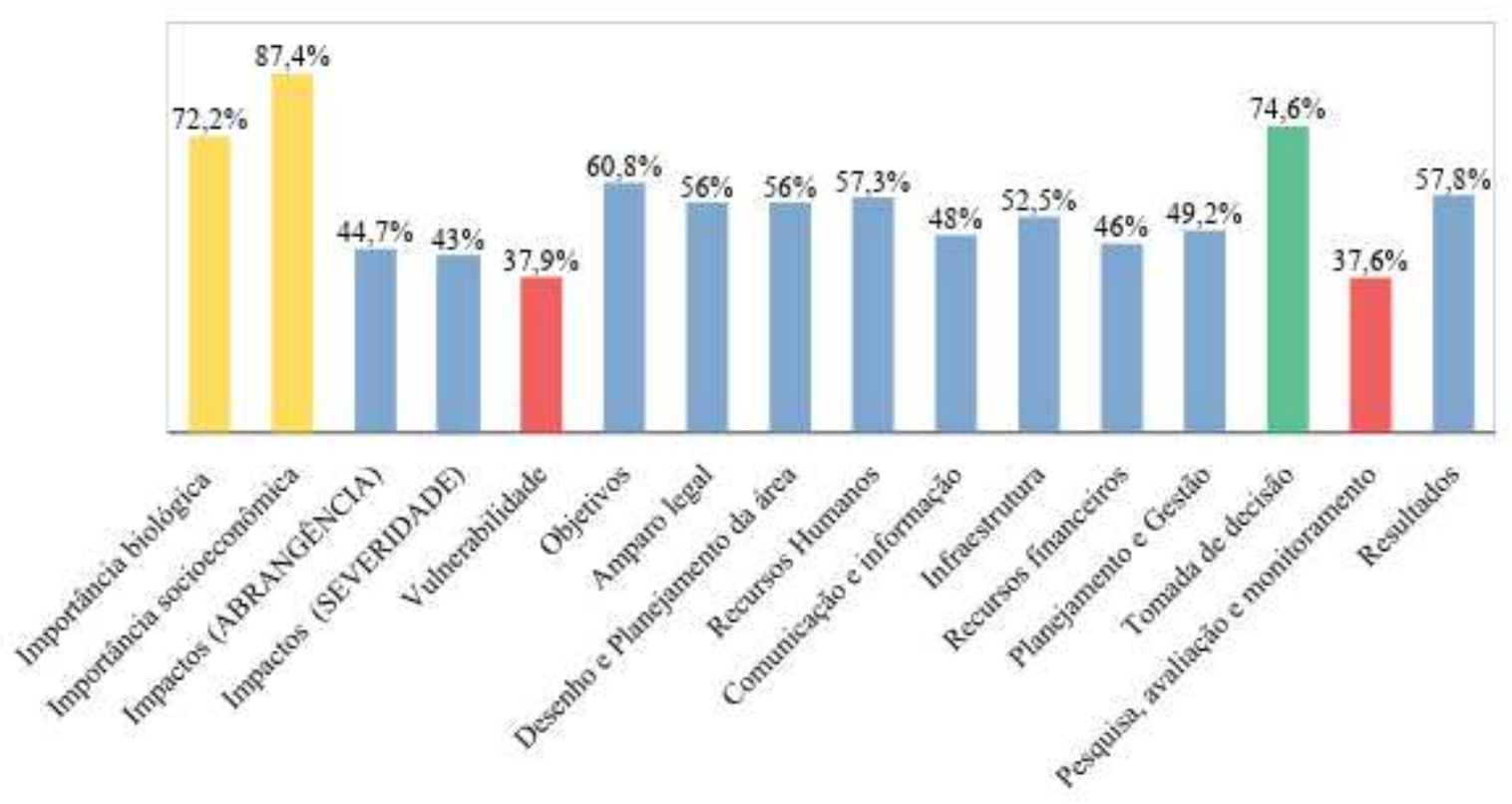

FIGURA 4 - Resultado de Efetividade por módulo. FONTE: Autores. 


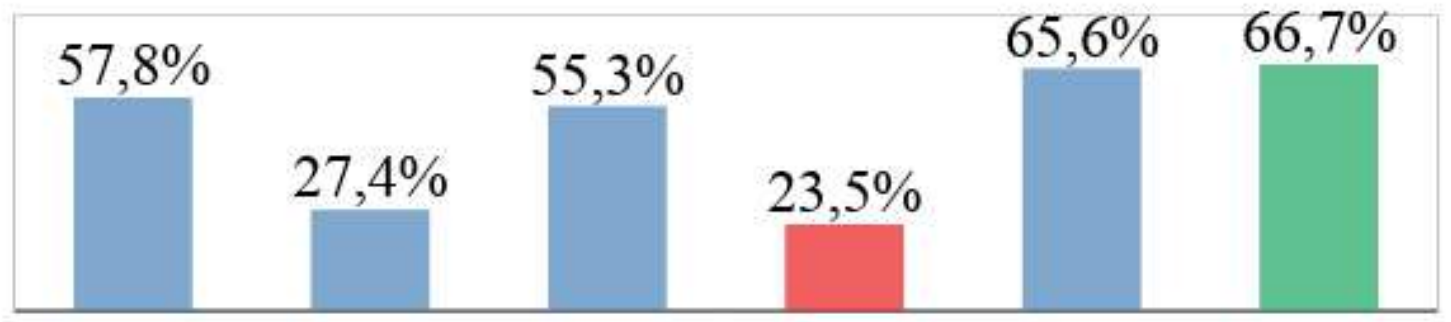

\section{ObservaçãoObservaçãoObservaçãoObservaçãoObservaçãoObservação 1 2 3 4 5 6}

FIGURA 5 - Resultado de efetividade por questionário.

FONTE: Autores

com $57,8 \%$ de efetividade. Se analisarmos as porcentagens de efetividade individualmente (Figura 5), percebem-se diferenças entre pontos de vista a respeito do processo de gestão na APABF. No módulo "Planejamento e Gestão", enquanto alguns analistas responderam que sua avaliação alcançou $85 \%$ de efetividade, outros responderam que atingiu apenas $20 \%$.

A APABF, por meio do método utilizado, possui uma efetividade de gestão e conservação de $49,4 \%$. Segundo este dado, a efetividade de gestão e de conservação dela é minimamente satisfatória, ou seja, a área protegida tem alguns recursos fundamentais para a gestão, mas minimamente aceitáveis. Nesse contexto, a APABF não está cumprindo com os objetivos de conservação e se encontra altamente vulnerável. Comparando com os resultados obtidos em 2006, quando o ICMBio realizou a avaliação de todas as UCs Federais com o uso do método RAPPAM, a APABF apresentou uma efetividade de gestão de 47\%. Em 2010, em um novo ciclo de avaliação, a efetividade da APABF foi para $52 \%$ (ICMBio, 2012). Dessa forma, esses três resultados não representam uma diferença significativa, indicando que, depois da primeira avaliação em 2006, a APABF ainda não avançou em termos de gestão, segundo os dados do método utilizado.

Infere-se que a efetividade da APABF pode ser melhorada se pelo menos sete módulos, dos 14 analisados, obtiverem pontuação acima de $50 \%$. Os módulos "Vulnerabilidade" e "Pesquisa, Avaliação e Monitoramento" necessitam de maior atenção por parte da equipe gestora. É importante notar os dados sobre abrangência e severidade dos impactos sobre a APABF, pois eles evidenciam a ampla gama de impactos provenientes das mais diversas atividades. A agricultura, a pesca, a infraestrutura, as pressões urbanas, a poluição das águas fluviais e o tráfego de embarcações foram considerados como de alto impacto sobre os ecossistemas naturais. Já a silvicultura, que se desenvolveu de forma expressiva nos 15 anos analisados no mapeamento realizado em 2015 (Prestes, 2016), foi considerada de médio impacto, tal como a invasão por espécies exóticas vegetais. O questionário indicou um índice médio sobre as estratégias para enfrentar ameaças e 
pressões na APABF, dado que sugere que algumas ações devem ser realizadas sobre esse aspecto, pois existe uma diversidade de conflitos e pressões de uso bastante extensa verificada nos módulos 3 e 4 . Esses resultados levantam reflexões essenciais para a equipe da APABF sobre a forma de gestão desenvolvida e a percepção da própria equipe da UC.

\section{Conclusão}

O método proposto possibilitou verificar a qualidade de gestão realizada na APABF, bem como as pressões de uso sobre a mesma, de modo que foi possível observar os pontos fortes e fracos da gestão, dando condição para que o órgão competente interceda sobre os aspectos deficitários.

A metodologia desenvolvida abrange elementos passíveis para as APAs, como a diversidade de usos e impactos, integração ou não com demais instrumentos de gestão e aspectos de participação social. A capacidade de adaptação do método é outro ponto positivo a se considerar. Os módulos sobre impactos no meio biofísico, por exemplo, podem ser ajustados de acordo com o alvo de conservação da APA. Da mesma forma, se destaca a facilidade na obtenção do percentual de efetividade e seu cenário correspondente por meio da atribuição de escores das respostas. Também se avalia como importante disponibilizar a opção de resposta "Desconheço". No caso do teste realizado na APABF, esta opção foi bastante utilizada (146 vezes) e possibilita evidenciar a desinformação sobre determinado elemento de gestão.

Devido à diversidade de sistemas ambientais e às particularidades de uso do território das APAs, avaliações mais abrangentes são necessá- rias para intervenções decisivas na gestão. Isto é, não se excluem estudos mais aprofundados para o aprimoramento da gestão. Também é igualmente importante estimar o ponto de vista do conselho gestor sobre a efetividade de gestão da unidade de conservação. Nesse caso, embora a aplicação demande mais tempo, a metodologia é viável de ser aplicada para o conselho gestor da APA somente se forem realizadas oficinas para nivelamento de informações entre os participantes. Infere-se também que a escala de escores pode ser aperfeiçoada para melhor representar a realidade.

Considerando os dados expostos, conclui-se que o método utilizado atendeu a expectativa de avaliação rápida de eficiência de gestão da APABF. Além disso, os indicadores presentes nele têm como base metodologias que já foram amplamente testadas e o framework desenvolvido pela IUCN.

\section{Referências}

Bensusan, N. Conservação da biodiversidade em áreas protegidas. Rio de Janeiro: FGV, 2006.

Brasil. Lei $n^{\circ}$ 6.902, de 27 de abril de 1981. Dispõe sobre a criação de Estações Ecológicas, Áreas de Proteção Ambiental e dá outras providências. Brasília: DOU de 28/4/1981.

Brasil. Lei $n^{\circ}$ 9.985, de 9 de julho de 2000. Regulamenta o art. 225, $\S 1^{\circ}$, incisos I, II, III e VII da Constituição Federal, institui o Sistema Nacional de Unidades de Conservação da Natureza e dá outras providências. Brasília: DOU de 19/7/2000.

Brasil. Decreto $n^{\circ} 4.340$, de 22 de agosto de 2002. Regulamenta artigos da Lei $\mathrm{n}^{\circ} 9.985$, de 18 de julho de 2000, que dispõe sobre o Sistema Nacional de Unidades de Conservação da Natureza - SNUC, e dá outras providências. Brasília: DOU de 23/8/2002.

Cabral, N. R. A. J.; Souza, M. P. de. Area de proteção am- 
biental: planejamento e gestão de paisagens protegidas. 2 . ed. São Carlos: RIMA, 2005.

Cifuentes, M.; Izurieta A.; Faria, H. H. de. Medición de la efectividad del manejo de areas protegidas. Turrialba: WWF e IUCN, 2000. Disponível em: <https://portals.IUCN.org/ library/efiles/documents/2000-131-Es.pdf $>$. Acesso em: mar. 2016.

CONAMA - Conselho Nacional do Meio Ambiente. Resolução $n^{\circ} 10$, de 14 de dezembro de 1988. Brasília: DOU de $11 / 08 / 1989$.

CONAMA - Conselho Nacional do Meio Ambiente. Resolução $n^{\circ} 428$, de 17 de dezembro de 2010. Brasília: DOU de 20/12/2010.

Dourojeanni, M. J.; Pádua, M. T. J. Biodiversidade: a hora decisiva. Curitiba: UFPR, 2007.

Ervin, J. Rapid Assessment and Prioritization of Protected Area Management (Rapppam) Methodology. Gland: WWF. 2003. Disponível em: <assets.panda.org/downloads/rappam. pdf $>$. Acesso em: fev. 2016.

Faria, H. H. de. Eficácia de gestão de unidades de conservação gerenciadas pelo Instituto Florestal de São Paulo, Brasil. Presidente Prudente, Tese (Doutorado em Geografia) - UNESP, 2004.

Faria H. H. de; Pires, A. S. Administração, manejo ou gestão de unidades de conservação? Revista Fórum Ambiental da Alta Paulista, 8(3), 43-59. 2012. doi: 10.17271/19800827

Gidsicki, D. Protocolo de avaliação de efetividade de gestão de mosaicos de áreas protegidas no Brasil. Manaus, Dissertação (Mestrado em Gestão de Áreas Protegidas na Amazônia) - INPA, 2012.

Guerra, A. J. T.; Coelho, M. C. N. Unidades de conservação: abordagens e características geográficas. Rio de Janeiro: Bertrand Brasil, 2009.

Guimarães E.; Pellin A. BiodiverCidade: desafios e oportunidades na gestão de áreas protegidas urbanas. São Paulo: Matrix, 2015.

Hockings, M.; Stolton, S.; Leverington, F.; Dudley, N.; Courrau, J. Evaluating Effectiveness: A framework for assessing management effectiveness of protected areas. 2 . ed. Gland and Cambridge: IUCN, 2006. Disponível em: $<$ https://portals.IUCN.org/library/efiles/documents/pag014.pdf>. Acesso em: fev. 2016.

ICMBio - Instituto Chico Mendes de Conservação da Biodiversidade. Avaliação comparada das aplicações do método Rappam nas unidades de conservação federais, nos ciclos 2005-06 e 2010. 2012. Disponível em: <http://www. icmbio.gov.br/portal/images/stories/comunicacao/downloads/relatrio\%20rappam $\% 202005 \% 20 x \% 202010 \% 20-\% 20$ verso\%20integral.pdf $>$. Acesso em: fev. 2016.

Leverington, F.; Costa, K. L.; Courrau, J.; Pavese, H.; Nolte, C.; Marr, M.; Coad, L.; Burgess, N.; Bomhard, B.; Hockings, M. Management effectiveness evaluation in protected areas: a global study. 2. ed. Brisbane: The University of Queensland, 2010. Disponível em: $<\mathrm{http} / /$ www.eci.ox.ac. $\mathrm{uk} /$ publications/downloads/coad11-protected-areas.pdf $>$. Acesso em: fev. 2016.

Leverington, F.; Hockings, M.; Costa, K. L. Management effectiveness evaluation in protected areas: Report for the project Global study into management effectiveness evaluation of protected areas. Gatton: The University of Queensland, IUCN, TNC, WWF, 2008a. Disponível em: $<$ http://cmsdata.IUCN.org/downloads/evaluationpas08. pdf $>$. Acesso em: fev. 2016.

Leverington, F.; Hockings, M.; Pavese, H. Costa, K. L.; Courrau, J. Management effectiveness evaluation in protected areas: Global study. Supplementary report $N^{\circ}$. 1: Overview of approaches and methodologies. 2008b. Gatton: The University of Queensland, IUCN, TNC, WWF. Disponível em: $<$ http://cmsdata.IUCN.org/downloads/methodologyreportdec08_final.pdf>. Acesso em: fev. 2016.

MMA - Ministério do Meio Ambiente. Cadastro Nacional de Unidades de Conservação. Disponível em: $<\mathrm{http}$ ://www. mma.gov.br/areas-protegidas/cadastro-nacional-de-ucs/ dados-consolidados $>$. Acesso em: abr. 2017.

MMA - Ministério do Meio Ambiente. Panorama da conservação dos ecossistemas costeiros e marinhos no Brasil, 2010. Disponível em: <http://www.mma.gov.br/estruturas/205/publicacao/205_publicacao03022011100749. pdf $>$. Acesso em: fev. 2015.

Oliveira, D. de. Avaliação de efetividade de gestão de 
Unidades de Conservação: mosaico do Apuí - Amazonas/ AM. Brasília, Dissertação (Mestrado em Política e Gestão Ambiental) - UnB, 2012.

Orth, D.; Debetir, E. Unidades de conservação: gestão e conflitos. Florianópolis: Insular, 2007.

Prestes, L. D. Áreas de Proteção Ambiental (APA): conservação e sustentabilidade - o caso da APA da Baleia Franca (SC/Brasil). Porto Alegre, Dissertação (Mestrado em Geografia) - UFRGS, 2016.

Pureza, F.; Pellin A.; Pádua, C. Unidades de Conservação. São Paulo: Matrix, 2015.
Ribeiro, S. C.; Barbosa, C. S. Limont, M. Avaliação da efetividade de gestão participativa na APA da Serra da Mantiqueira. Revista Geo UERJ, 22, 92-107, 2010. doi: 10.12957/geouerj.2010.1460

Rodrigues, J. E. R. Sistema Nacional de Unidades de Conservação. São Paulo: Ed. Revista dos Tribunais, 2005.

Souza, J. V. C. Congresso Mundiais de Parques Nacionais da UICN (1962- 2003): registros e reflexões sobre o surgimento de um novo paradigma para a conservação da natureza. Brasília, Dissertação (Mestrado em Desenvolvimento Sustentável) - UnB, 2013. 